
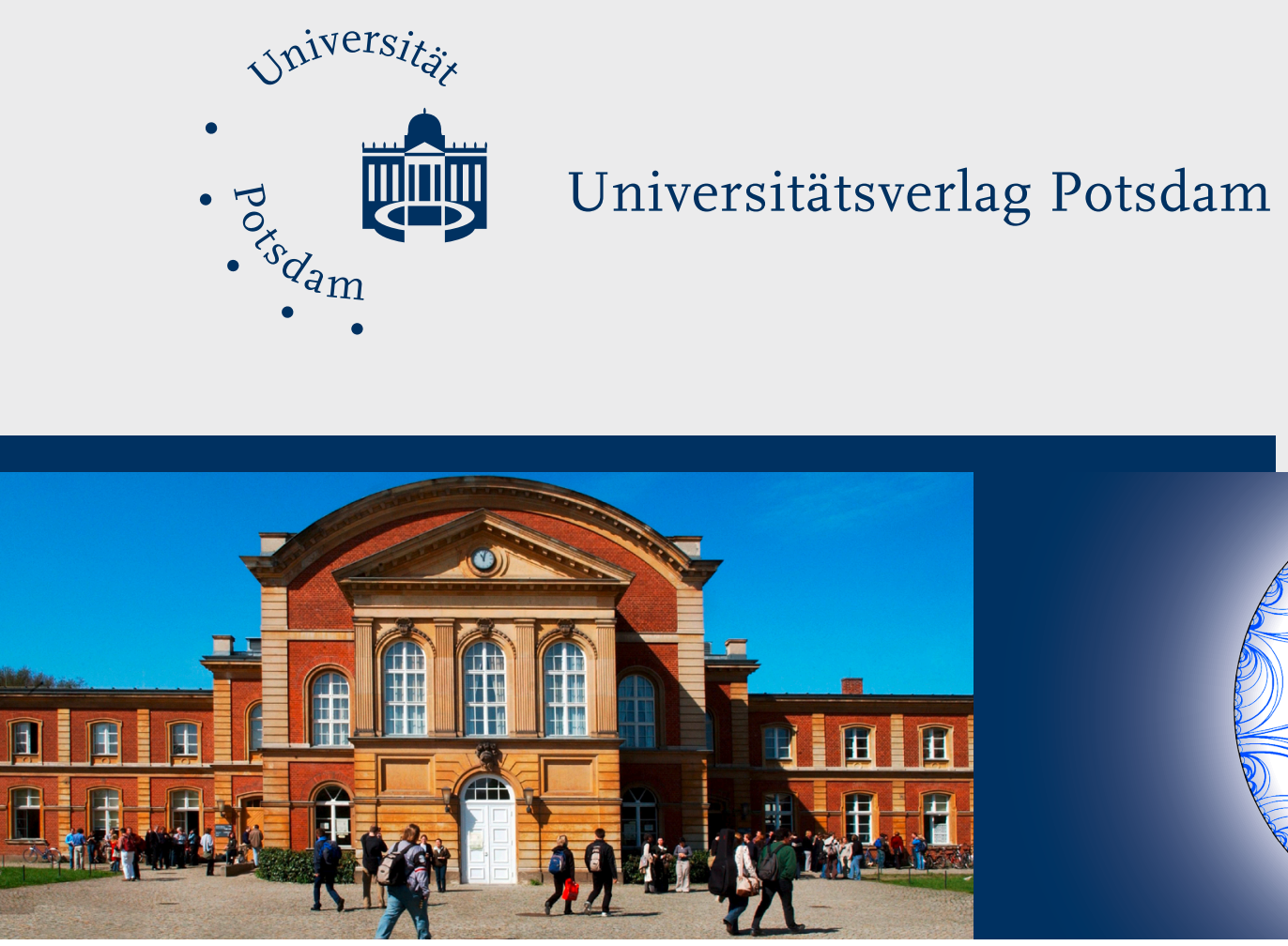

K. O. Makhmudov| O. I. Makhmudov| N. Tarkhanov

\title{
A Nonstandard Cauchy Problem for the Heat Equation
}

Preprints des Instituts für Mathematik der Universität Potsdam 4 (2015) 11 

Preprints des Instituts für Mathematik der Universität Potsdam 

Preprints des Instituts für Mathematik der Universität Potsdam $4(2015) 11$

K. O. Makhmudov | O. I. Makhmudov | N. Tarkhanov A Nonstandard Cauchy Problem for the Heat Equation 


\section{Bibliografische Information der Deutschen Nationalbibliothek}

Die Deutsche Nationalbibliothek verzeichnet diese Publikation in der Deutschen Nationalbibliografie; detaillierte bibliografische Daten sind im Internet über http://dnb.dnb.de abrufbar.

Universitätsverlag Potsdam 2015

http://verlag.ub.uni-potsdam.de/

Am Neuen Palais 10, 14469 Potsdam

Tel.: +49 (0)3319772533 / Fax: 2292

E-Mail: verlag@uni-potsdam.de

Die Schriftenreihe Preprints des Instituts für Mathematik der Universität Potsdam wird herausgegeben vom Institut für Mathematik der Universität Potsdam.

ISSN (online) 2193-6943

Kontakt:

Institut für Mathematik

Am Neuen Palais 10

14469 Potsdam

Tel.: +49 (0)331977 1028

WWW: http://www.math.uni-potsdam.de

Titelabbildungen:

1. Karla Fritze | Institutsgebäude auf dem Campus Neues Palais

2. Nicolas Curien, Wendelin Werner | Random hyperbolic triangulation

Published at: http://arxiv.org/abs/1105.5089

Das Manuskript ist urheberrechtlich geschützt.

Online veröffentlicht auf dem Publikationsserver der Universität Potsdam URN urn:nbn:de:kobv:517-opus4-83830

http://nbn-resolving.de/urn:nbn:de:kobv:517-opus4-83830 


\title{
A NONSTANDARD CAUCHY PROBLEM FOR THE HEAT EQUATION
}

\author{
K. O. MAKHMUDOV, O. I. MAKHMUDOV, AND N. TARKHANOV
}

\begin{abstract}
We consider a Cauchy problem for the heat equation in a cylinder $\mathcal{C}_{T}=\mathcal{X} \times(0, T)$ over a domain $\mathcal{X}$ in $\mathbb{R}^{n}$ with data on a strip lying on the lateral surface. The strip is of the form $S \times(0, T)$, where $S$ is an open subset of the boundary of $\mathcal{X}$. The problem is ill-posed. Under natural restrictions on the configuration of $S$ we derive an explicit formula for solutions of this problem.
\end{abstract}

\section{Contents}

1. Introduction 1

2. A Cauchy problem for parabolic equations 3

3. The heat equation in dimension one 5

4. An example of instability $\quad 7$

$\begin{array}{ll}\text { 5. A fundamental lemma } & 7\end{array}$

6. Heat conductivity in a cap type domain 11

7. Solvability criteria for the Cauchy problem 13

$\begin{array}{ll}\text { References } & 14\end{array}$

\section{INTRODUCTION}

By an ill-posed problem is meant any problem where the inverse operator is not continuous. If treated in Fréchet spaces, a problem is ill-posed if and only if it fails to be solvable for all data. Ill-posed problems still remain a challenge for researchers.

An advertising slogan related to the Cauchy problem for elliptic equations is that, after J. Hadamard, the Cauchy problem for the Laplace equation is ill-posed. Of course, the instability refers here to a standard setting, for in spaces with twonorm convergence the Cauchy problem for elliptic equations proves to be stable, see [AT90].

The character of instability, solvability criteria and regularisation methods of the Cauchy problem for elliptic equations are studied in [Tar95]. For the complete bibliography we refer the reader to this work. Much of the theory developed in [Tar95] extends immediately to other ill-posed problems of complex analysis or partial differential equations.

Date: November 12, 2015

2010 Mathematics Subject Classification. Primary 35K05; Secondary 35K15.

Key words and phrases. Heat equation, Cauchy problem, Carleman formulas. 
This paper is motivated by a problem posed by M. M. Lavrent'ev in the early 1980s, see for instance [LRS80]. It was to find an explicit formula for the temperature inside a plane domain by using partial lateral and initial data. This problem is solved in [Ike09].

The paper [PS12] gives a necessary and sufficient solvability condition for the Cauchy problem for the heat equation with partial lateral and initial conditions. However, it falls short of providing an explicit regularisation formula for the solution.

Our approach to the Cauchy problem for parabolic equations or systems in a cylinder $\mathcal{C}_{T}=\mathcal{X} \times(0, T)$ over a domain $\mathcal{X} \subset \mathbb{R}^{n}$ with partial lateral and initial data originates from [AV64]. More precisely, if one applies the Fourier or Laplace transform in the time variable $t \geq 0$ to a parabolic equation, it transforms to a family of elliptic equations with complex parameter $\tau$ in the domain $\mathcal{X}$. On transforming the data on the lateral boundary of $\mathcal{C}_{T}$ in a similar way we arrive at a family of Cauchy problems for an elliptic equation with parameter in $\mathcal{X}$ with data on a nonempty open subset $S$ of $\partial \mathcal{X}$. To study this problem one can apply immediately the constructive techniques elaborated in [Tar95]. It then remains to apply the inverse Laplace transform.

The Fourier transform is an important method of studying several problems of mathematical physics and differential equations. In the classical papers the functions under transform were assumed to be integrable at infinity. In physics one often applied the Fourier transform to slowly growing functions without sufficient substantiation. A strong mathematical theory of the Fourier transform for slowly growing functions was first elaborated in [Boc32]. No systematic study of the Fourier transform of rapidly growing functions, such that $e^{x}, e^{x^{2}}$, etc., had been done until [GS53] elaborated the theory of Fourier transform for functions growing arbitrarily rapidly. The generalisation of the Fourier transform to rapidly growing functions is well motivated. As but one example we mention that for the heat equation $u_{t}^{\prime}=u_{x x}^{\prime \prime}$ the natural domain, in which the Cauchy problem is solvable and has a unique solution, is constituted by all functions which grow more slowly than $e^{x^{2}}$, as $|x| \rightarrow \infty$, for each fixed $t \geq 0$. Note that a characteristic feature of the paper [GS53] is exit to a complex domain and considering functionals on classes of (usually, entire) analytic functions.

We examine the approach for applicability in the Cauchy problem for the heat equation in the cylinder over a cap type domain $\mathcal{X}$ in $\mathbb{R}^{3}$. After making the Laplace transformation, we get a Cauchy problem for the Helmholtz equation in $\mathcal{X}$ with data away a boundary piece lying in the plane $\left\{x_{3}=0\right\}$. The potential of the Helmholtz equation just amounts to $\imath \tau$. An explicit Carleman function for this problem was first described in [Yar77]. However, no unobjectionable proof had been done until [Ike01] revised the formula of [Yar77] in the case of Laplace equation. It was first [Yar04] where Sh. Yarmukhamedov completed his construction announced in [Yar77]. The paper [YY03] contains a particular construction of Carleman function for the Cauchy problem for the Helmholtz equation in a cap type domain in three dimensions.

Note that our approach applies to the Cauchy problem for the time-dependent system of couple-stress elasticity [MNT08] and Maxwell equations [MMT11] with partial lateral and initial data. 


\section{A CAUChy PRoblem for PARABolic EQUATIONS}

Let $\mathcal{X}$ be a bounded domain with smooth boundary in $\mathbb{R}^{n}$ and $S$ a nonempty open piece of the boundary surface $\partial \mathcal{X}$. We consider the Cauchy problem for the heat equation in the cylinder $\mathcal{C}_{T}=\mathcal{X} \times(0, T)$ with data on the strip $S \times(0, T)$ of the lateral surface of the cylinder, where $T>0$ is a fixed number. More precisely, given functions $f$ on $\mathcal{C}_{T}$ and $u_{0}, u_{1}$ on $S \times(0, T)$, find a function $u$ in $\mathcal{C}_{T}$ which satisfies

$$
\begin{aligned}
u_{t}^{\prime}=\Delta u+f & \text { in } \mathcal{C}_{T}, \\
u=u_{0} & \text { at } S \times(0, T), \\
u_{\nu}^{\prime}=u_{1} & \text { at } S \times(0, T),
\end{aligned}
$$

where $\nu$ is the unit outward normal vector for $\partial \mathcal{X}$, see Fig. 1.

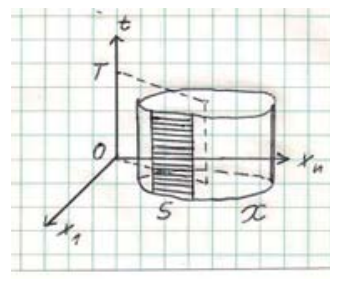

FIG. 1. A Cauchy problem for a parabolic equation in a cylinder with data on the lateral surface.

From results of [LO74] it follows that problem (2.1) has at most one smooth solution $u$.

The lateral surface does not contain any characteristic point for the heat equation, and so the Cauchy-Kovalevskaya theorem applies to problem (2.1). Hence, if moreover $S$ is really analytic, then for all real analytic data $f$ and $u_{0}, u_{1}$ this problem has locally close to $S \times(0, T)$ a real analytic solution $u$. By the above, it is unique.

In order to construct an approximate solution of the Cauchy problem (2.1) we continue the data $f$ and $u_{0}, u_{1}$ to the whole semicylinder $t>0$, i.e., $T=\infty$ in the sequel. On applying the Laplace transform in $t \in(0, \infty)$ to all equations of problem $(2.1)$

$$
\hat{u}(x, \tau):=\mathcal{L} u(x, \tau)=\int_{0}^{\infty} e^{-\imath \tau t} u(x, t) d t
$$

we get the family of Cauchy problems

$$
\begin{aligned}
(\Delta-\imath \tau) \hat{u}(\cdot, \tau) & =-u(\cdot, 0)-\hat{f}(\cdot, \tau) & & \text { in } \mathcal{X}, \\
\hat{u}(\cdot, \tau) & =\hat{u}_{0}(\cdot, \tau) & & \text { at } S, \\
\hat{u}_{\nu}^{\prime}(\cdot, \tau) & =\hat{u}_{1}(\cdot, \tau) & & \text { at } S
\end{aligned}
$$

in the domain $\mathcal{X}$, parametrised by the complex parameter $\tau$ running over a horizontal line in the lower half-plane. For any fixed $\tau$, one readily specifies $(2.2)$ as the Cauchy problem for the Helmholtz equation in $\mathcal{X}$ with data on a boundary piece $S$. 
This problem is well known to be unstable in the natural setting unless $S$ coincides with the whole boundary. This gives an evidence to the fact that problem (2.1) is ill-posed, see [LRS80], [PS12].

Denote by $\hat{G}(x ; \tau)$ the fundamental solution of convolution type for the Helmholtz operator $\Delta-\imath \tau$ in $\mathbb{R}^{n}$. (This is actually the convolution kernel of the resolvent $(\Delta-\imath \tau)^{-1}$.) The techniques developed in [Tar95] enable one to construct an approximate solution of problem (2.2). To wit, one finds a sequence of kernels $\hat{C}_{N}(x, y ; \tau)$, for $N=1,2, \ldots$, which differ from $\hat{G}(x-y ; \tau)$ by a smooth solution of the Helmholtz equation in $y \in \mathcal{X}$ and whose Cauchy data in $y$ tend to zero at $\partial \mathcal{X} \backslash S$. Then, for fixed $\tau$, an approximate solution of (2.2) is obtained from the so-called Carleman formula

$\hat{u}(x, \tau)=\lim _{N \rightarrow \infty}\left(-\int_{S}\left(\hat{C}_{N}(x, \cdot ; \tau) \partial_{\nu} \hat{u}-\partial_{\nu} \hat{C}_{N}(x, \cdot ; \tau) \hat{u}\right) d s+\int_{\mathcal{X}} \hat{C}_{N}(x, \cdot ; \tau)(\Delta-\imath \tau) \hat{u} d y\right)$

for all $x \in \mathcal{X}$, where $d s$ is the surface measure on $\partial \mathcal{X}$.

Assume that the inverse Laplace transform is possible under the limit passage in the last formula. By the familiar convolution theorem for the Laplace transform, we get

$$
\begin{aligned}
\mathcal{L}^{-1}\left(\hat{C}_{N}(x, \cdot ; \tau) \partial_{\nu} \hat{u}\right) & =\mathcal{L}^{-1}\left(\mathcal{L}\left(\mathcal{L}^{-1} \hat{C}_{N}(x, \cdot ; \tau)\right) \mathcal{L}\left(\partial_{\nu} u\right)\right) \\
& =\mathcal{L}^{-1} \hat{C}_{N}(x, \cdot ; \tau) * \partial_{\nu} u \\
& =\int_{0}^{t}\left(\mathcal{L}^{-1} \hat{C}_{N}\right)\left(x, \cdot ; t-t^{\prime}\right) \partial_{\nu} u\left(\cdot, t^{\prime}\right) d t^{\prime}
\end{aligned}
$$

Similarly,

$$
\mathcal{L}^{-1}\left(\partial_{\nu} \hat{C}_{N}(x, \cdot ; \tau) \hat{u}\right)=\int_{0}^{t} \partial_{\nu}\left(\mathcal{L}^{-1} \hat{C}_{N}\right)\left(x, \cdot ; t-t^{\prime}\right) u\left(\cdot, t^{\prime}\right) d t^{\prime}
$$

and

$$
\begin{aligned}
& \mathcal{L}^{-1}\left(\hat{C}_{N}(x, \cdot ; \tau)(\Delta-\imath \tau) \hat{u}\right) \\
& \quad=\int_{0}^{t}\left(\mathcal{L}^{-1} \hat{C}_{N}\right)\left(x, \cdot ; t-t^{\prime}\right)\left(\Delta-\partial_{t^{\prime}}\right) u\left(\cdot, t^{\prime}\right) d t^{\prime}-\left(\mathcal{L}^{-1} \hat{C}_{N}\right)(x, \cdot ; t) u(\cdot, 0) .
\end{aligned}
$$

Hence, applying the inverse Laplace transform to both sides of equality (2.3) we arrive at the formula

$$
\begin{aligned}
u(x, t)=\lim _{N \rightarrow \infty}( & -\int_{\mathcal{X}}\left(\mathcal{L}^{-1} \hat{C}_{N}\right)(x, \cdot ; t) u(\cdot, 0) d y \\
& -\int_{S} \int_{0}^{t}\left(\left(\mathcal{L}^{-1} \hat{C}_{N}\right)\left(x, \cdot ; t-t^{\prime}\right) \partial_{\nu} u-\partial_{\nu}\left(\mathcal{L}^{-1} \hat{C}_{N}\right)\left(x, \cdot ; t-t^{\prime}\right) u\right) d s d t^{\prime} \\
& \left.+\int_{\mathcal{X}} \int_{0}^{t}\left(\mathcal{L}^{-1} \hat{C}_{N}\right)\left(x, \cdot ; t-t^{\prime}\right)\left(\Delta-\partial_{t^{\prime}}\right) u d y d t^{\prime}\right)
\end{aligned}
$$

whenever $(x, t) \in \mathcal{C}_{T}$.

Set

$$
C_{N}(x, y ; t)=\mathcal{L}^{-1} \hat{C}_{N}(x, y ; \tau)=\frac{1}{2 \pi} \int_{\Im \tau=\gamma} e^{\imath t \tau} \hat{C}_{N}(x, y ; \tau) d \tau
$$


for $(x, y)$ away from the diagonal in $\mathcal{X} \times \overline{\mathcal{X}}$ and for $t>0$, where $\gamma$ is a sufficiently small negative number. A direct calculation shows that

$$
\begin{aligned}
\left(\Delta_{y}-\partial_{t}\right) C_{N}(x, y ; t) & =\frac{1}{2 \pi} \int_{\Im \tau=\gamma} e^{\imath t \tau}\left(\Delta_{y}-\imath \tau\right) \hat{C}_{N}(x, y ; \tau) d \tau \\
& =0
\end{aligned}
$$

for $(x, y)$ and $t$ in the domain of $C_{N}$. Moreover, $C_{N}(x, y ; t)$ tends to zero in certain sense in $y$ away from $S$ on the boundary of $\mathcal{X}$, as $N \rightarrow \infty$. Hence, the sequence of kernels $C_{N}(x, y ; t)$ generalises to problem $(2.1)$ the concept of Carleman function in the Cauchy problem for elliptic equations developed in [Tar95]. If any Carleman function $C_{N}$ is given explicitly, one easily obtains a formula for an approximate solution of (2.1).

Lemma 2.1. If $C_{N}(x, y ; t)$ is a Carleman function of problem (2.1), then the formula

$$
u_{N}=\mathcal{P}_{i, N}(u(\cdot, 0))+\mathcal{P}_{s, N}\left(u_{1}\right)+\mathcal{P}_{d, N}\left(u_{0}\right)+\mathcal{P}_{v, N}(f)
$$

gives an approximate solution of the problem in the cylinder $\mathcal{C}_{T}$, where

$$
\begin{array}{rlrl}
\mathcal{P}_{i, N}(u(\cdot, 0)) & =-\int_{\mathcal{X}} C_{N}(x, \cdot ; t) u(\cdot, 0) d y, & \mathcal{P}_{s, N}\left(u_{1}\right)=-\iint_{S 0}^{t} C_{N}\left(x, \cdot ; t-t^{\prime}\right) u_{1} d s d t^{\prime}, \\
\mathcal{P}_{d, N}\left(u_{0}\right)=\iint_{S 0}^{t} \int_{\nu} C_{N}\left(x, \cdot ; t-t^{\prime}\right) u_{0} d s d t^{\prime}, & \mathcal{P}_{v, N}(f)=-\iint_{\mathcal{X} 0} C_{N}\left(x, \cdot ; t-t^{\prime}\right) f d y d t^{\prime} .
\end{array}
$$

Note that the values $u(x, 0)$ at the cylinder bottom are uniquely determined by the values $u(x, t)$ for small $t>0$, provided that $u$ bears some continuity up to $t=0$. Therefore, $u(x, 0)$ are determined uniquely from the values of $u$ and $u_{\nu}^{\prime}$ on the strip $S \times(0, T)$. However, the Carleman formulas are most simple if they include the initial values $u(x, 0)$.

While the method of approximate solution of problem (2.1) described in this section extends to more general parabolic equations, we treat it in detail for the particular case $n=3$.

\section{The heat EQuation in Dimension one}

The case $n=1$ is not emphatic to demonstrate the approach, for the initial value problem is stable for ordinary differential equations. However, explicit formulas available in this case provide an insight to elucidate the general ill-posed problem, too.

Let $\mathcal{X}=(a, b)$ be a finite interval on the real axis. Choose $S=\{a\}$ and consider the Cauchy problem of finding a function $u$ in the rectangle $\mathcal{C}_{T}=\mathcal{X} \times(0, T)$ satisfying

$$
\begin{aligned}
u_{t}^{\prime}(x, t) & =u_{x x}^{\prime \prime}(x, t)+f(x, t) & & \text { for } \quad(x, t) \in \mathcal{C}_{T}, \\
u(a, t) & =u_{0}(t) & & \text { for } t \in(0, T), \\
u_{x}^{\prime}(a, t) & =u_{1}(t) & & \text { for } t \in(0, T),
\end{aligned}
$$

where $f$ and $u_{0}, u_{1}$ are given functions on $\mathcal{C}_{T}$ and $(0, T)$, respectively. 
On converting this problem by Laplace transform we obtain the family of initial value problems

$$
\begin{aligned}
\hat{u}_{x x}^{\prime \prime}(x, \tau)-\imath \tau \hat{u}(x, \tau) & =-u(x, 0)-\hat{f}(x, \tau) \text { for } x \in \mathcal{X}, \\
\hat{u}(a, \tau) & =\hat{u}_{0}(\tau) \\
\hat{u}_{x}^{\prime}(a, \tau) & =\hat{u}_{1}(\tau)
\end{aligned}
$$

on the interval $\mathcal{X}$, parametrised by a complex parameter $\tau$ running over a horizontal line $\Im \tau=\gamma$. Looking for a solution of the form $\hat{u}(x, \tau)=c \exp \lambda x$ to the homogeneous equation we get the characteristic equation $\lambda^{2}-\imath \tau=0$ for $\lambda$, whose roots are

$$
\lambda_{ \pm}(\tau)= \pm \sqrt{|\tau|} e^{\imath \frac{\arg \tau}{2}} \frac{1+\imath}{\sqrt{2}}
$$

An elementary verification now shows that the unique solution of initial value problem (3.1) is given by

$$
\hat{u}(x, \tau)=\hat{c}_{+}(x, \tau) e^{\lambda_{+}(\tau) x}+\hat{c}_{-}(x, \tau) e^{\lambda_{-}(\tau) x},
$$

where

$$
\hat{c}_{ \pm}(x, \tau)=\frac{1}{2} \frac{e^{\lambda_{\mp}(\tau) a}}{\lambda_{\mp}(\tau)}\left(\lambda_{\mp}(\tau) \hat{u}_{0}(\tau)-\hat{u}_{1}(\tau)\right)+\int_{a}^{x} \frac{1}{2} \frac{e^{\lambda_{\mp}(\tau) y}}{\lambda_{\mp}(\tau)}(u(y, 0)+\hat{f}(y, \tau)) d y .
$$

This can be rewritten in the form

$$
\hat{u}(x, \tau)=\left(\hat{C}(x, a ; \tau) \hat{u}_{1}-\partial_{y} \hat{C}(x, a ; \tau) \hat{u}_{0}\right)+\int_{\mathcal{X}} \hat{C}(x, y ; \tau)(-u(y, 0)-\hat{f}(y, \tau)) d y
$$

with

$$
\hat{C}(x, y ; \tau)= \begin{cases}\frac{\sinh \lambda_{-}(\tau)(x-y)}{\lambda_{-}(\tau)}, & \text { if } \quad x-y \geq 0 \\ 0, & \text { if } \quad x-y<0 .\end{cases}
$$

Note that (3.2) substitutes precisely for Carleman formula (2.3), since $\partial_{\nu}=-\partial_{y}$, as is easy to see.

It remains to apply the inverse Laplace transform to both sides of equality (3.2), thus obtaining

$$
\begin{aligned}
u(x, t)= & -\int_{\mathcal{X}}\left(\mathcal{L}^{-1} \hat{C}\right)(x, y ; t) u(y, 0) d y \\
& +\int_{0}^{t}\left(\left(\mathcal{L}^{-1} \hat{C}\right)\left(x, a ; t-t^{\prime}\right) u_{1}^{\prime}\left(t^{\prime}\right)-\partial_{y}\left(\mathcal{L}^{-1} \hat{C}\right)\left(x, a ; t-t^{\prime}\right) u_{0}\left(t^{\prime}\right)\right) d t^{\prime} \\
& -\int_{\mathcal{X}} \int_{0}^{t}\left(\mathcal{L}^{-1} \hat{C}\right)\left(x, y ; t-t^{\prime}\right) f\left(y, t^{\prime}\right) d y d t^{\prime}
\end{aligned}
$$

for all $(x, t) \in \mathcal{C}_{T}$. We have used the convolution theorem for the Laplace transform, as in the proof of $(2.4)$. Obviously, the kernel $\left(\mathcal{L}^{-1} \hat{C}\right)(x, y ; t)$ vanishes if $x-y<0$ or $t<0$. Since

it follows that

$$
\mathcal{L}^{-1}\left(\frac{e^{-\sqrt{\imath \tau}(x-y)}}{\sqrt{\imath \tau}}\right)=\frac{1}{\sqrt{\pi t}} e^{-\frac{(x-y)^{2}}{4 t}},
$$

$$
\left(\mathcal{L}^{-1} \hat{C}\right)(x, y ; t)=-\frac{1}{\sqrt{4 \pi t}} e^{-\frac{(x-y)^{2}}{4 t}}+\frac{1}{2} \mathcal{L}^{-1}\left(\frac{e^{\sqrt{\imath \tau}(x-y)}}{\sqrt{\imath \tau}}\right)
$$


for $x-y \geq 0$ and $t>0$, the inverse Laplace transform being interpreted in the sense of [GS53]. The first summand on the right-hand side just amounts to the fundamental solution of the operator $\partial_{x x}^{2}-\partial_{t}$.

\section{An eXAmple of instability}

The Cauchy problem (2.1) displays certain character of instability for all $n \geq 1$. We show an example following to [LRS80] and [PS12]. Let $\mathcal{X}$ be a bounded domain with smooth boundary in the half-space $\left\{x_{n}>0\right\}$ of $\mathbb{R}^{n}$, whose boundary contains a nonempty open piece $S$ of the hyperplane $\left\{x_{n}=0\right\}$. Fix a natural number $N$ and consider the sequence of functions

$$
u_{k}(x, t)=\frac{\exp \left(k^{2}(t-T)+k x_{n}\right)}{k^{N}}
$$

which are infinitely differentiable in the closure of the cylinder $\mathcal{C}_{T}=\mathcal{X} \times(0, T)$, where $T>0$.

A trivial verification shows that

$$
\left(u_{k}\right)_{t}^{\prime}=\Delta u_{k}
$$

in $\mathcal{C}_{T}$. Moreover,

and

$$
u_{k}(x, 0)=\frac{\exp \left(-k^{2} T+k x_{n}\right)}{k^{N}}
$$

$$
\begin{aligned}
u_{k}(x, t) & =\frac{\exp \left(k^{2}(t-T)\right)}{k^{N}}, \\
\left(u_{k}\right)_{x_{n}}^{\prime}(x, t) & =\frac{\exp \left(k^{2}(t-T)\right)}{k^{N-1}}
\end{aligned}
$$

at the lateral strip $S \times(0, T)$. Hence it follows that

$$
u_{k}(x, 0) \rightarrow 0
$$

in the topology of $C^{\infty}(\overline{\mathcal{X}})$ and

$$
\begin{aligned}
u_{k}(x, t) & \rightarrow 0, \\
\left(u_{k}\right)_{x_{n}}^{\prime}(x, t) & \rightarrow 0
\end{aligned}
$$

in the norm of $C^{s}(\bar{S} \times[0, T])$, provided that $N>2 s+1$.

However,

$$
u_{k}(x, T)=\frac{\exp \left(k x_{n}\right)}{k^{N}} \rightarrow \infty
$$

as $k \rightarrow \infty$, if $x_{n}>0$. Hence, the solution of problem (2.1) fails to depend continuously on the data.

\section{A FUNDAMENTAL LEMMA}

Let $\sigma$ be a positive number. Consider the entire function $K(w)=\exp \left(\sigma w^{2}\right)$ of complex variable $w \in \mathbb{C}$. The restriction of $K$ to any vertical line $w=u_{0}+\imath v$ just amounts to $K(u+\imath v)=K(u) \exp \left(2 \imath \sigma u v-\sigma v^{2}\right)$, which is a rapidly decreasing function of $v$.

Given two different points $x=\left(x^{\prime}, x_{3}\right)$ and $y=\left(y^{\prime}, y_{3}\right)$ in $\mathbb{R}^{3}$, set $r^{\prime}=\left|y^{\prime}-x^{\prime}\right|$ and introduce the integral

$$
\Phi(x, y)=\frac{-1}{2 \pi^{2}} \frac{1}{K\left(x_{3}\right)} \int_{0}^{\infty} \Im\left(\frac{K(w)}{w-x_{3}}\right) \frac{\cos \lambda \vartheta}{\sqrt{r^{\prime 2}+\vartheta^{2}}} d \vartheta,
$$


where $w=y_{3}+\imath \sqrt{r^{\prime 2}+\vartheta^{2}}$ and $\lambda$ is a complex parameter. On separating the imaginary part we get

$$
\Phi(x, y)=\int_{0}^{\infty} k(x, y ; \vartheta) \cos \lambda \vartheta d \vartheta
$$

with $k(x, y ; \vartheta)$ given by

$$
\frac{-1}{2 \pi^{2}} \frac{e^{\sigma\left(y_{3}^{2}-x_{3}^{2}\right)} e^{-\sigma\left(r^{\prime 2}+\vartheta^{2}\right)}}{\vartheta^{2}+r^{2}}\left(\left(y_{3}-x_{3}\right) \frac{\sin 2 \sigma y_{3} \sqrt{r^{\prime 2}+\vartheta^{2}}}{\sqrt{r^{\prime 2}+\vartheta^{2}}}-\cos 2 \sigma y_{3} \sqrt{r^{\prime 2}+\vartheta^{2}}\right) .
$$

The convergence of the improper integral on the right-hand side of (5.1) is thus guaranteed by the factor $e^{-\sigma \vartheta^{2}}$.

Lemma 5.1. As defined by equality (5.1), the function $\Phi(x, y)$ is represented in the form

$$
\Phi(x, y)=\frac{-1}{4 \pi} \frac{e^{-\lambda r}}{r}+R(x, y),
$$

where $R(x, y)$ is a twice continuously differentiable function of the variable $y \in \mathbb{R}^{3}$ including the point $y=x$.

Proof. Divide the interval of integration in (5.1) into two parts, namely $[0,1)$ and $[1, \infty)$. Write $I_{1}(x, y)$ and $I_{2}(x, y)$ for the first and the second integral, respectively. Since $\sqrt{r^{\prime 2}+\vartheta^{2}} \geq 1$ for the second integral, $I_{2}(x, y)$ is a $C^{\infty}$ function of $y \in \mathbb{R}^{n}$ including the point $y=x$.

The first integral is transformed as

$$
\begin{aligned}
I_{1}(x, y) & =\frac{-1}{2 \pi^{2}} \frac{1}{K\left(x_{3}\right)} \int_{0}^{1} \Im\left(\frac{K(w)-K\left(x_{3}\right)}{w-x_{3}}\right) \frac{\cos \lambda \vartheta}{\sqrt{r^{\prime 2}+\vartheta^{2}}} d \vartheta \\
& +\frac{-1}{2 \pi^{2}} \int_{0}^{1} \Im\left(\frac{1}{w-x_{3}}\right) \frac{\cos \lambda \vartheta}{\sqrt{r^{\prime 2}+\vartheta^{2}}} d \vartheta,
\end{aligned}
$$

where

$$
\Im\left(\frac{1}{w-x_{3}}\right)=-\frac{\sqrt{r^{\prime 2}+\vartheta^{2}}}{\vartheta^{2}+r^{2}} .
$$

Since the entire function $Q(w)=\frac{K(w)-K\left(x_{3}\right)}{w-x_{3}}$ is real for real $w$, it follows from the expansion

$$
\frac{\Im Q\left(y_{3}+\imath \sqrt{r^{\prime 2}+\vartheta^{2}}\right)}{\sqrt{r^{\prime 2}+\vartheta^{2}}}=\sum_{j=0}^{\infty}(-1)^{j} \frac{Q^{(j)}\left(y_{3}\right)}{(2 j+1) !}\left(r^{\prime 2}+\vartheta^{2}\right)^{j}
$$

that the first term on the right-hand side of (5.3) is a $C^{\infty}$ function of $x$ and $y$. The second term reduces to

$$
\begin{aligned}
& \int_{0}^{1} \frac{\cos \lambda \vartheta}{\vartheta^{2}+r^{2}} d \vartheta=\sum_{j=0}^{\infty}(-1)^{j} \frac{\lambda^{2 j}}{(2 j) !} \int_{0}^{1} \frac{\vartheta^{2 j}}{\vartheta^{2}+r^{2}} d \vartheta \\
& \quad=\int_{0}^{1} \frac{1}{\vartheta^{2}+r^{2}} d \vartheta-\frac{\lambda^{2}}{2} \int_{0}^{1} \frac{\vartheta^{2}}{\vartheta^{2}+r^{2}} d \vartheta+\sum_{j=2}^{\infty}(-1)^{j} \frac{\lambda^{2 j}}{(2 j) !} \int_{0}^{1} \frac{\vartheta^{2 j}}{\vartheta^{2}+r^{2}} d \vartheta
\end{aligned}
$$


up to the factor $1 / 2 \pi^{2}$. The first integral on the right-hand side is equal to

$$
-\frac{\pi}{2} \frac{1}{r}-\int_{1}^{\infty} \frac{1}{\vartheta^{2}+r^{2}} d \vartheta
$$

and the second integral just amounts to

$$
-\frac{\lambda^{2}}{2}\left(1-\int_{0}^{1} \frac{r^{2}}{\vartheta^{2}+r^{2}} d \vartheta\right)=-\frac{\lambda^{2}}{2}+\frac{\lambda^{2} r^{2}}{2}\left(-\frac{\pi}{2} \frac{1}{r}-\int_{1}^{\infty} \frac{1}{\vartheta^{2}+r^{2}} d \vartheta\right) .
$$

Therefore, the second term on the right-hand side of (5.3) has the form

$-\frac{\pi}{2} \frac{1}{r}\left(1+\frac{\lambda^{2} r^{2}}{2}\right)-\frac{\lambda^{2}}{2}-\left(1+\frac{\lambda^{2} r^{2}}{2}\right) \int_{1}^{\infty} \frac{1}{\vartheta^{2}+r^{2}} d \vartheta+\sum_{j=2}^{\infty}(-1)^{j} \frac{\lambda^{2 j}}{(2 j) !} \int_{0}^{1} \frac{\vartheta^{2 j}}{\vartheta^{2}+r^{2}} d \vartheta$

up to the factor $1 / 2 \pi^{2}$. Since

$$
1+\frac{\lambda^{2} r^{2}}{2}=e^{-\lambda r}+\lambda r-\sum_{j=3}^{\infty}(-1)^{j} \frac{(\lambda r)^{j}}{j !}
$$

we conclude readily that

$$
I_{1}(x, y)=\frac{-1}{4 \pi} \frac{e^{-\lambda r}}{r}+R_{1}(x, y)
$$

where $R_{1}(x, y)$ is a twice continuously differentiable function with respect to the variable $y$ including the point $y=x$. Setting $R=I_{1}+R_{1}$, we arrive at formula (5.2).

Lemma 5.2. As defined in (5.1), the function $\Phi(x, y)$ satisfies the Helmholtz equation $\Delta \Phi-\lambda^{2} \Phi=0$ in $y \in \mathbb{R}^{3} \backslash\{x\}$.

Proof. Denote

$$
Q(w)=\frac{-1}{2 \pi^{2}} \frac{1}{K\left(x_{3}\right)} \frac{K(w)}{w-x_{3}},
$$

where $w=y_{3}+\imath \sqrt{r^{\prime 2}+\vartheta^{2}}$. Then

$$
\begin{aligned}
\Delta_{y} \Phi(x, y) & =\int_{0}^{\infty} \Delta_{y}\left(\frac{\Im Q(w)}{\sqrt{r^{\prime 2}+\vartheta^{2}}}\right) \cos \lambda \vartheta d \vartheta \\
& =\frac{1}{2 \imath} \int_{0}^{\infty} \Delta_{y}\left(\frac{Q(w)-Q(\bar{w})}{\sqrt{r^{\prime 2}+\vartheta^{2}}}\right) \cos \lambda \vartheta d \vartheta
\end{aligned}
$$

The function under the Laplace operator $\Delta_{y}$ is of the form $f\left(Y, y_{n}\right)$, where $Y=\left|y^{\prime}-x^{\prime}\right|^{2}$. A simple verification shows that

$$
\Delta_{y} f\left(Y, y_{n}\right)=4 Y f_{Y Y}^{\prime \prime}+2(n-1) f_{Y}^{\prime}+f_{y_{n} y_{n}}^{\prime \prime},
$$

the partial differential operator on the right-hand side of this equality will be denoted by $\Delta_{Y, y_{n}}$. Therefore, we shall have established the lemma if we prove that

$$
\begin{aligned}
& \int_{0}^{\infty} \Delta_{Y, y_{3}}\left(\frac{Q(w)}{\sqrt{Y+\vartheta^{2}}}\right) \cos \lambda \vartheta d \vartheta=\lambda^{2} \int_{0}^{\infty} \frac{Q(w)}{\sqrt{Y+\vartheta^{2}}} \cos \lambda \vartheta d \vartheta \\
& \int_{0}^{\infty} \Delta_{Y, y_{3}}\left(\frac{Q(\bar{w})}{\sqrt{Y+\vartheta^{2}}}\right) \cos \lambda \vartheta d \vartheta=\lambda^{2} \int_{0}^{\infty} \frac{Q(\bar{w})}{\sqrt{Y+\vartheta^{2}}} \cos \lambda \vartheta d \vartheta
\end{aligned}
$$

for $y \neq x$. 
We prove mainly the first equality of (5.5). To this end we denote the left-hand side of this equality by $I$. Using (5.4) one obtains

$$
\begin{aligned}
I & :=\int_{0}^{\infty} \Delta_{Y, y_{3}}\left(\frac{Q(w)}{\sqrt{Y+\vartheta^{2}}}\right) \cos \lambda \vartheta d \vartheta \\
& =\int_{0}^{\infty}\left(4 Y\left(\frac{Q(w)}{\sqrt{Y+\vartheta^{2}}}\right)_{Y Y}^{\prime \prime}+4\left(\frac{Q(w)}{\sqrt{Y+\vartheta^{2}}}\right)_{Y}^{\prime}+\left(\frac{Q(w)}{\sqrt{Y+\vartheta^{2}}}\right)_{y_{3} y_{3}}^{\prime \prime}\right) \cos \lambda \vartheta d \vartheta
\end{aligned}
$$

and a trivial calculation yields

$$
\begin{aligned}
4\left(\frac{Q(w)}{\sqrt{Y+\vartheta^{2}}}\right)_{Y}^{\prime} & =2\left(\frac{\imath Q^{\prime}(w)}{Y+\vartheta^{2}}-\frac{Q(w)}{\left(Y+\vartheta^{2}\right)^{3 / 2}}\right) \\
4 Y\left(\frac{Q(w)}{\sqrt{Y+\vartheta^{2}}}\right)_{Y Y}^{\prime \prime} & =Y\left(\frac{-Q^{\prime \prime}(w)}{\left(Y+\vartheta^{2}\right)^{3 / 2}}-\frac{3 \imath Q^{\prime}(w)}{\left(Y+\vartheta^{2}\right)^{2}}+\frac{3 Q}{\left(Y+\vartheta^{2}\right)^{5 / 2}}\right), \\
\left(\frac{Q(w)}{\sqrt{Y+\vartheta^{2}}}\right)_{y_{3} y_{3}}^{\prime \prime} & =\frac{Q^{\prime \prime}(w)}{\sqrt{Y+\vartheta^{2}}} .
\end{aligned}
$$

On grouping the multipliers of the same derivatives of $Q$ we get $I=I_{1}+I_{2}+I_{3}$, where

Since

$$
\begin{aligned}
I_{1} & :=\int_{0}^{\infty} \frac{\vartheta^{2}}{\left(Y+\vartheta^{2}\right)^{3 / 2}} Q^{\prime \prime}(w) \cos \lambda \vartheta d \vartheta \\
I_{2} & :=\int_{0}^{\infty} \frac{2 \vartheta^{2}-Y}{\left(Y+\vartheta^{2}\right)^{2}} \imath Q^{\prime}(w) \cos \lambda \vartheta d \vartheta \\
I_{3} & :=\int_{0}^{\infty} \frac{Y-2 \vartheta^{2}}{\left(Y+\vartheta^{2}\right)^{5 / 2}} Q(w) \cos \lambda \vartheta d \vartheta
\end{aligned}
$$

$$
d_{\vartheta} Q^{\prime}(w)=Q^{\prime \prime}(w) \frac{\imath \vartheta}{\sqrt{Y+\vartheta^{2}}} d \vartheta
$$

then, integrating the first integral by parts, we obtain

$$
I_{1}=\int_{0}^{\infty}\left(\frac{Y-\vartheta^{2}}{\left(Y+\vartheta^{2}\right)^{2}} \cos \lambda \vartheta-\frac{\lambda \vartheta}{Y+\vartheta^{2}} \sin \lambda \vartheta\right) \imath Q^{\prime}(w) d \vartheta
$$

whence

$$
I_{1}+I_{2}=\int_{0}^{\infty} \frac{\vartheta^{2}}{\left(Y+\vartheta^{2}\right)^{2}} \imath Q^{\prime}(w) \cos \lambda \vartheta d \vartheta-\int_{0}^{\infty} \frac{\lambda \vartheta}{Y+\vartheta^{2}} \imath Q^{\prime}(w) \sin \lambda \vartheta d \vartheta
$$

In both integrals on the right-hand side here we integrate once again by parts using the equality

Then, the first integral transforms to

$$
d_{\vartheta} Q(w)=Q^{\prime}(w) \frac{\imath \vartheta}{\sqrt{Y+\vartheta^{2}}} d \vartheta .
$$

$$
-\int_{0}^{\infty}\left(\left(\frac{1}{\left(Y+\vartheta^{2}\right)^{3 / 2}}-\frac{3 \vartheta^{2}}{\left(Y+\vartheta^{2}\right)^{5 / 2}}\right) \cos \lambda \vartheta-\frac{\lambda \vartheta}{\left(Y+\vartheta^{2}\right)^{3 / 2}} \sin \lambda \vartheta\right) Q(w) d \vartheta
$$

and the second one to

Thus,

$$
\int_{0}^{\infty}\left(-\frac{\lambda \vartheta}{\left(Y+\vartheta^{2}\right)^{3 / 2}} \sin \lambda \vartheta+\frac{\lambda^{2}}{\sqrt{Y+\vartheta^{2}}} \cos \lambda \vartheta\right) Q(w) d \vartheta
$$

$$
I_{1}+I_{2}=-\int_{0}^{\infty} \frac{Y-2 \vartheta^{2}}{\left(Y+\vartheta^{2}\right)^{5 / 2}} Q(w) \cos \lambda \vartheta d \vartheta+\lambda^{2} \int_{0}^{\infty} \frac{Q(w)}{\sqrt{Y+\vartheta^{2}}} \cos \lambda \vartheta d \vartheta
$$


Since the first integral on the right-hand side just amounts to $I_{3}$, it follows that

$$
I=\lambda^{2} \int_{0}^{\infty} \frac{Q(w)}{\sqrt{Y+\vartheta^{2}}} \cos \lambda \vartheta d \vartheta
$$

as desired.

Finally, the function $Q(\bar{w})$ is obtained from $Q(w)$ by substituting $-\imath$ for $\imath$. Therefore, the calculations do not change and the second equality of (5.5) follows as well.

For $\lambda=0$, the results of this section are still valid with $K(w)=\exp (\sigma w)$, where $\sigma>0$, see [Ike01, Ike09].

\section{Heat CONDUCtivity in a CAP TYPE Domain}

Assume that $\mathcal{X}$ is a bounded domain in the upper half-space $\left\{x_{3}>0\right\}$ of $\mathbb{R}^{3}$ whose boundary consists of a smooth surface $S$ lying in the half-space $\left\{x_{3}>0\right\}$, and a closed piece of the plane $\left\{x_{3}=0\right\}$. Such domains are usually referred to as cap type domains. Note that the unit outward normal vector on the piece $\partial \mathcal{X} \backslash S$ just amounts to $(0,0,-1)$.

Following (5.1) we introduce

$$
\hat{C}_{\sigma}(x, y ; \tau)=\frac{-1}{2 \pi^{2}} \frac{1}{K\left(x_{3}\right)} \int_{0}^{\infty} \Im\left(\frac{K(w)}{w-x_{3}}\right) \frac{\cos (\sqrt{\imath \tau} \vartheta)}{\sqrt{r^{\prime 2}+\vartheta^{2}}} d \vartheta
$$

where $w=y_{3}+\imath \sqrt{r^{\prime 2}+\vartheta^{2}}$ and $\tau$ is a complex parameter. An easy calculation shows that

$$
\hat{C}_{\sigma}(x, y ; \tau)=\int_{0}^{\infty} k_{\sigma}(x, y ; \vartheta) \cos (\sqrt{\imath \tau} \vartheta) d \vartheta
$$

where $k_{\sigma}(x, y ; \vartheta)$ is given by

$$
\frac{-1}{2 \pi^{2}} \frac{e^{\sigma\left(y_{3}^{2}-x_{3}^{2}\right)} e^{-\sigma\left(r^{\prime 2}+\vartheta^{2}\right)}}{\vartheta^{2}+r^{2}}\left(\left(y_{3}-x_{3}\right) \frac{\sin 2 \sigma y_{3} \sqrt{r^{\prime 2}+\vartheta^{2}}}{\sqrt{r^{\prime 2}+\vartheta^{2}}}-\cos 2 \sigma y_{3} \sqrt{r^{\prime 2}+\vartheta^{2}}\right) .
$$

Hence it follows that

$$
\begin{aligned}
k_{\sigma}(x, y ; \vartheta) & =\frac{1}{2 \pi^{2}} \frac{e^{-\sigma x_{3}^{2}} e^{-\sigma\left(r^{\prime 2}+\vartheta^{2}\right)}}{\vartheta^{2}+r^{2}} \\
\partial_{y_{3}} k_{\sigma}(x, y ; \vartheta) & =\frac{1}{\pi^{2}} \frac{e^{-\sigma x_{3}^{2}} e^{-\sigma\left(r^{\prime 2}+\vartheta^{2}\right)}}{\left(\vartheta^{2}+r^{2}\right)^{2}} x_{3}\left(1+\sigma\left(\vartheta^{2}+r^{2}\right)\right)
\end{aligned}
$$

on the plane $y_{3}=0$. On applying Lemma 5.2 we conclude that $\hat{C}_{\sigma}(x, y ; \tau)$ is a Carleman function of Cauchy problem (2.2) in the domain $\mathcal{X}$ with data on $S$, parametrised by $\tau$.

Therefore, the theory of Section 2 applies to the Cauchy problem for the heat equation in $\mathcal{C}_{T}=\mathcal{X} \times(0, T)$ with data on $S \times(0, T)$. It remains to evaluate the inverse Laplace transform

$$
C_{\sigma}(x, y ; t)=\mathcal{L}^{-1} \hat{C}_{\sigma}(x, y ; \tau)=\int_{0}^{\infty} k_{\sigma}(x, y ; \vartheta) \mathcal{L}^{-1} \cos (\sqrt{\imath \tau} \vartheta) d \vartheta
$$

of $\hat{C}_{\sigma}(x, y ; \tau)$, which reduces to evaluating the inverse Laplace transform of the function $\cos (\sqrt{\imath \tau} \vartheta)$, i.e.,

$$
\mathcal{L}^{-1} \cos (\sqrt{\imath \tau} \vartheta)=\frac{1}{2 \pi \imath} \int_{\Re p=-\gamma} e^{t p} \cos (\sqrt{p} \vartheta) d p=\frac{1}{\vartheta^{2}}\left(\mathcal{L}^{-1} \cos \sqrt{s}\right)\left(\frac{t}{\vartheta^{2}}\right)
$$


for $\vartheta>0$.

It would be desirable to explicitly evaluate the inverse Laplace transform of $\cos \sqrt{s}$ but we have not been able to do this. The computer algebra system Maple gives no answer to this question, for $\cos \sqrt{s}$ fails to be the Laplace transform of a function on the nonnegative semiaxis satisfying $|f(t)| \leq C e^{k t}$ for $t \geq 0$. The formal approach does not yield any substantial formula. On formally extending the equality

$$
\mathcal{L}^{-1} \exp (-a \sqrt{s})=\frac{a}{\sqrt{4 \pi t^{3}}} \exp \left(-\frac{a^{2}}{4 t}\right),
$$

which is valid for $a>0$, analytically to $a= \pm \imath$ and using the Euler formula we obtain

$$
\mathcal{L}^{-1} \cos \sqrt{s}=\frac{1}{2}\left(\frac{-\imath}{\sqrt{4 \pi t^{3}}} \exp \frac{1}{4 t}+\frac{\imath}{\sqrt{4 \pi t^{3}}} \exp \frac{1}{4 t}\right)=0,
$$

a nonsense. As is often the case, if the Laplace transform $F=\mathcal{L} f$ expands as

$$
F(s)=s^{p} \sum_{n=0}^{\infty} c_{n} s^{r n}
$$

where $r>0$ is a rational number, then

$$
f(t)=\frac{1}{t^{p+1}} \sum_{n=0}^{\infty} \frac{c_{n}}{\Gamma(-p-r n)} t^{-r n}
$$

for $t>0$. For the function $F(s)=\cos \sqrt{s}$ this formal argument leads immediately to the formula

$$
f(t)=\frac{1}{t} \sum_{n=0}^{\infty} \frac{c_{n}}{\Gamma(-n / 2)} t^{-n / 2}
$$

where $c_{n}=(-1)^{n / 2} / n$ ! for even $n$ and $c_{n}=0$ for odd $n$. Since $\Gamma(z)$ has poles at all points $z=0,-1, \ldots$, we recover once again that $f \equiv 0$. We thus conclude that the inverse Laplace transform of $\cos \sqrt{s}$ should be interpreted within the sophisticated theory of [GS53].

Theorem 6.1. Let $\mathcal{X}$ be a cap type domain in $\mathbb{R}^{3}$. Then, for any $u \in H^{2,1}\left(\mathcal{C}_{T}\right)$, it follows that

$$
\begin{aligned}
u(x, t)=\lim _{\sigma \rightarrow \infty}( & -\int_{\mathcal{X}} C_{\sigma}(x, \cdot ; t) u(\cdot, 0) d y \\
& -\int_{S} \int_{0}^{t}\left(C_{\sigma}\left(x, \cdot ; t-t^{\prime}\right) \partial_{\nu} u-\partial_{\nu} C_{\sigma}\left(x, \cdot ; t-t^{\prime}\right) u\right) d s d t^{\prime} \\
& \left.+\int_{\mathcal{X}} \int_{0}^{t} C_{\sigma}\left(x, \cdot ; t-t^{\prime}\right)\left(\Delta-\partial_{t^{\prime}}\right) u d y d t^{\prime}\right)
\end{aligned}
$$

whenever $(x, t) \in \mathcal{C}_{T}$.

Proof. This is a direct consequence of formula (6.1) and the approach developed in Section 2. 


\section{Solvability CRiteria FOr the CAUChy PROBlem}

When regularising an ill-posed problem one usually assumes a priori that there a solution to the problem with appropriate properties. It would be desirable that the convergence of a numerical solution of the ill-posed problem with given data be evidence of the existence of a solution while the divergence would testify to the nonexistence. This is similar to the fact that an a priori proof of the stability of a correct problem often implies an existence theorem. Perhaps one can think of the limit of regularisations, if there is any, as a generalised solution of the problem, for it is a tested substitute for the solution, if there is no real solution. We now clarify this concept.

If $u \in H^{2,1}\left(\mathcal{C}_{T}\right)$ is a solution of Cauchy problem (2.1) then Theorem 6.1 implies that

$$
\begin{aligned}
u(x, t)=\lim _{\sigma \rightarrow \infty}( & -\int_{\mathcal{X}} C_{\sigma}(x, \cdot ; t) u(\cdot, 0) d y \\
& -\int_{S} \int_{0}^{t}\left(C_{\sigma}\left(x, \cdot ; t-t^{\prime}\right) u_{1}-\partial_{\nu} C_{\sigma}\left(x, \cdot ; t-t^{\prime}\right) u_{0}\right) d s d t^{\prime} \\
& \left.-\int_{\mathcal{X}} \int_{0}^{t} C_{\sigma}\left(x, \cdot ; t-t^{\prime}\right) f d y d t^{\prime}\right)
\end{aligned}
$$

for all $(x, t) \in \mathcal{C}_{T}$.

Conversely, assume that, given data $f$ and $u_{0}, u_{1}$, the limit on the right-hand side of (7.1) exists at each point $(x, t) \in \mathcal{C}_{T}$ and thus defines a function $u$ in the cylinder $\mathcal{C}_{T}$. One may ask if $u$ satisfies the Cauchy problem (2.1). The affirmative answer would allow one to formulate a solvability criterion for problem (2.1), cf. for instance [Yar04]. While being adequately motivated from the viewpoint of mathematics, such a criterion is efficient by no means. Hence, the right-hand side of (7.1) may be regarded as approximate solution of problem (2.1) in any case. The mathematical problem consists most likely in specifying the sense in which this formula is optimal.

Acknowledgments The research of the second author was supported by the Deutscher Akademischer Austauschdienst. The authors are greatly indepted to Prof. V. Stepanenko for several helpful comments concerning the generalised Laplace transform. 


\section{REFERENCES}

[AV64] Agranovich, M. S., and Vishik, M. I., Elliptic problems with a parameter and parabolic problems of general type, Uspekhi Mat. Nauk 19 (1964), Issue 3, 53-161.

[AT90] Aizenberg, L. A., and Tarkhanov, N. N., Conditionally stable problems and Carleman formulas, Siberian Math. J. 31 (1990), no. 6, 9-15.

[Boc32] Bochner, S., Vorlesungen über Fouriersche Integrale, Akademie Verlag, Leipzig, 1932.

[Doe50] Doetsch, G., Handbuch der Laplace-Transformation, Bde I-III, Birkhäuser, Basel, Switzerland, 1950.

[GS53] Gelfand, I. M., and Shilov, G. E., The Fourier transform of rapidly increasing functions und uniqueness of the Cauchy problem, Uspekhi Mat. Nauk 8 (1953), Issue 6 (53), 3-54.

[Ike01] Ikehata, M., Inverse conductivity problem in the infinite slab, Inverse Problems $\mathbf{1 7}$ (2001), 437-454.

[Ike09] Ikehata, M., Two analytical formulae of the temperature inside a body by using partial lateral and initial data, Inverse Problems 25 (2009), 035011 (21 pp).

[LO74] Landis, E. M., and Oleynik, O. A., Generalised analyticity and related properties of solutions of elliptic and parabolic equations, Uspekhi Mat. Nauk 29 (1974), no. 2, $190-215$.

[LRS80] Lavrent'ev, M. M., Romanov, V. G., and Shishatskii, S. P., Ill-Posed Problems of Mathematical Physics and Analysis, Nauka, Moscow, 1980.

[MNT08] Makmudov, O., Niyozov, I., and Tarkhanov, N., The Cauchy problem of couple-stress elasticity, In: Complex Analysis and Dynamical Systems III, Contemporary Mathematics, vol. 455, Amer. Math. Soc., Providence, RI, 2008, pp. 297-310.

[MMT11] Makhmudov, K., Makhmudov, O., and Tarkhanov, N., Equations of Maxwell type, J. Math. Anal. Appl. 378 (2011), Issue 1, 64-75.

[PS12] Puzyrev, R., and Shlapunov, A., On an ill-posed problem for the heat equation, J. of Siberian Federal Univ., Math. a Phys. 5 (2012), no. 3, 337-348.

[Tar95] Tarkhanov, N., The Cauchy Problem for Solutions of Elliptic Equations, Akademie Verlag, Berlin, 1995.

[Yar77] Yarmukhamedov, Sh., The Cauchy problem for the Laplace equation, Soviet Math. Dokl. 18 (1977), no. 4, 939-942.

[Yar04] Yarmukhamedov, Sh., The Carleman function and the Cauchy problem for the Laplace equation, Siberian Math. J. 45 (2004), no. 3, 580-595.

[YY03] Yarmukhamedov, Sh., and Yarmukhamedov, I., The Cauchy problem for the Helmholtz equation, In: Ill-posed and non-classical problems of mathematical physics and analysis, VSP, Utrecht, 2003, 143-172.

Department of Mechanics and Mathematics, University of Samarkand, University Boulevard 15, 140104 SAMARKand, UzBekistan

E-mail address: komil.84@mail.ru

Department of Mechanics and Mathematics, University of Samarkand, University Boulevard 15, 140104 Samarkand, UzBekistan

E-mail address: olimjan@yahoo.com

Institute of Mathematics, University of Potsdam, Karl-Liebknecht Street 24/25, 14476 Potsdam (Golm), Germany

E-mail address: tarkhanov@math.uni-potsdam.de 